\title{
Sprung in die zweite Reihe? Zu den lokalen Bedingungen rent gap-getriebener immobilien- wirtschaftlicher Aufwertung in Brandenburgs Mittelstädten
}

\section{Leapfrogging to the second row? On the local constellations of rent gap-driven real estate upgrading in Brandenburg's medium-sized towns}

https://doi.org/10.1515/zfw-2020-0008

Eingereicht: 09. März 2020; akzeptiert: 26. April 2021

Zusammenfassung: Gegenwärtig sind nicht mehr nur deutsche Großstädte von Immobilien- und Mietpreissteigerungen geprägt, sondern zunehmend auch die Wohnungsmärkte des metropolitanen Umlandes. Angesichts dieser Entwicklungen drängt sich die Frage auf, ob sich die Wohnungsfrage zunehmend auch auf Mittelstädte ausweitet. Der Beitrag geht dieser Frage mit Rückgriff auf die rent gap-Theorie nach. Diese versteht die Entwicklungen auf Immobilienmärkten nicht als abstrakte Automatismen der räumlichen Ausweitung immobilienwirtschaftlicher Aufwertung, sondern betont, dass die rent gap gesellschaftlich unter verschiedenen historisch-geographischen Bedingungen immer wieder neu hergestellt wird. Folglich diskutiert der Beitrag die konkreten lokalen Konstellationen aus angebots- und nachfrageseitigen Entwicklungen sowie stadtpolitischen Interventionen, die Grundrentensteigerungen ermöglichen. Als empirisches Beispiel dienen die brandenburgischen Zweite Reihe-Städte Fürstenwalde (Spree) und Neuruppin. Während beide Mittelstädte eine ähnliche Bevölkerungsentwicklung $\mathrm{zu}$ verzeichnen haben, sind sie verkehrsinfrastrukturell unterschiedlich gut an das Berliner Zentrum angebunden. Die Ergebnisse zeigen, dass sich in der besser an Berlin angeschlossenen Stadt Fürstenwalde (Spree) keine rent gap öffnet, während in der weiter entfernten Stadt Neu-

\footnotetext{
*Korrespondierender Autor: Dr. Michael Mießner, Alpen-AdriaUniversitat Klagenfurt, Institute of Geography and Regional Science, Lakesidepark, Klagenfurt 9020, Austria,

E-Mail: michael.miessner@aau.at

Richard Bůžek, Westfälische Wilhelms-Universität Münster, Institute of Geography, Heisenbergstr. 2, Munster, Nordrhein-Westfalen, Germany, E-Mail: rbuzek@uni-muenster.de
}

ruppin eine rent gap entsteht. Diese - den abstrakten Theorien räumlicher Ausbreitung von Immobilieninvestitionen entgegenstehenden - Forschungsergebnisse machen deutlich, dass die jeweiligen historisch-geographischen und lokalen Bedingungen entscheidend sind, um die Entstehung von rent gaps zu verstehen. Der Beitrag schlussfolgert, dass die Wirtschafts- und Stadtgeographie diesen Bedingungen größere Aufmerksamkeit widmen sollte, um Entwicklungen in Mittelstädten zu verstehen.

Schlagworte: rent gap, Gentrifzierung, Immobilienmarkt, Mittelstädte

Abstract: Currently, not only German metropolises are affected by increasing real estate prices and rents, but also housing markets in the metropolitan hinterlands. In light of these trends, the question occurs whether the housing question is expanding on medium-sized towns, too. This paper pursues this question by drawing on rent gap theory. That theory conceptualizes developments on real estate markets not as an abstract automatism of the spatial expansion of real estate-driven upgrading. Instead, rent gap theory highlights the continuous social production of rent gaps under diverse historical-geographic conditions. Hence, the paper discusses the concrete local constellations of supply- and demand-side developments as well as interventions of municipal politics which enable increasing ground rents. Empirical references are the case studies Fürstenwalde (Spree) and Neuruppin in Brandenburg's second row. Whereas both medium-sized towns are characterized by a similar population development, they differ regarding their transport connection to Berlin city center. The results indicate that in Fürstenwalde (Spree) no rent gap is opening despite its more advanta- 
geous connection to Berlin, while in the spatially more distant town of Neuruppin, a rent gap is occurring. These results contrast abstract theories of the spatial expansion of real estate investments and make clear, that the specific historical-geographic and local constellations matter in order to comprehend the emergence of rent gaps. Therefore, economic and urban geography should bring these rent gap constellations in smaller towns in greater focus.

Keywords: rent gap, gentrification, real estate market, medium-sized towns

\section{Einleitung}

Steigende Immobilienpreise und Mieten prägen gegenwärtig nicht mehr allein deutsche Großstädte, sondern erfassen auch die Wohnungsmärkte des metropolitanen Umlandes und einiger gut verflochtener Klein- und Mittelstädte, wie jüngste immobilienwirtschaftliche Studien verdeutlichen ( $F+B$ 2020). Damit drängt sich die Frage auf, ob sich die zuspitzende Wohnungsfrage mittlerweile nicht lediglich auf deutsche Metropolen beschränkt, sondern sich auch jenseits der urbanen Zentren zunehmend stellt.

Mit Bezug auf die wirtschaftsgeographische Finanzialisierungsforschung (z. B. Christophers 2012; Ouma und Bläser 2015; Scheuplein 2020) können diese Entwicklungen wie folgt erklärt werden: Im Zuge des finanzdominierten Akkumulationsregimes (Sablowski 2011; Zeller 2011) ist ein immer größerer Teil des Finanzkapitals, insbesondere seit der Wirtschafts- und Finanzkrise und der anschließenden Niedrigzinsphase - die sogenannte „wall of money“ (Fernandez und Aalbers 2016) - auf der Suche nach einer profitablen Anlagesphäre. Diese findet es unter anderem in deutschen Immobilien, die als stabile und lukrative Anlagemöglichkeit gelten, weshalb seitdem große Mengen von nationalem wie internationalem Finanzkapital (Mösgen und Schipper 2017; Wijburg und Aalbers 2017) in diesen „sekundären Kapitalkreislauf“ (Harvey 2006 [1982]) fließen. Dabei standen zunächst die wachsenden Metropolen wie Frankfurt am Main, Berlin, Hamburg, Köln oder München im Zentrum des Investitionsgeschehens (Heeg 2013; Schürt 2013). Jüngst stiegen auch die Immobilien- und Mietpreise in vielen kleineren Großstädten und in Mittelstädten (IVD 2018; Savills 2018). Folgt man der Finanzialisierungsforschung, könnte dieser Immobilien- und Mietpreisanstieg, neben anderen Ursachen, auf eine Ausbreitung der Investitionen entlang der Städte-Hierarchie (Lees 2006) zurückgeführt werden, bei welcher der finanzgetriebene Anlagedruck von den Met- ropolen in nachgeordnete Städte fließt (Fehlberg und Mießner 2015). Solche Platzierungen des Finanzkapitals in Wohnraum sind jedoch von der allgemeinen Renditesituation im Vergleich mit anderen Anlagesphären abhängig, sodass, neben politischen Regulierungen und der lokalen wirtschaftlichen Situation, eine ausreichende Transparenz zwischen den einzelnen Anlageklassen hergestellt werden muss (Ouma und Bläser 2015). Während diese Transparenz für die Metropolen mittlerweile weitgehend hergestellt ist (Bitterer und Heeg 2015; AK OGA 2019; F+B 2020), besteht noch eine relativ hohe Intransparenz bzgl. Wohnimmobilien in kleineren deutschen Städten. Diese ergibt sich aufgrund regionaler und sektoraler Teilmärkte sowie der Fragmentierung des Immobilienmarktes und der allgemeinen Charakterisierung von Immobilien als heterogene und autonome Güter (Musil 2019, S. 18; Rottke 2017, S. 87). Folglich sind trotz regulatorischer Öffnungen deutscher Grundstücks- und Immobilienmärkte für globale Kapitalströme (Heeg 2004) nicht alle Teilmärkte durch eine hohe Liquidität gekennzeichnet und Bestandteil eines global vergleichbaren modernen Portfoliomanagements (vgl. Vornholz 2015). Deshalb greift der Finanzialisierungsansatz für die Erklärung aktueller Prozesse der immobilienwirtschaftlichen Aufwertung in kleineren Städten zu kurz: Es kann aufgrund der Marktintransparenzen nicht von einem automatischen Überschwappen von Investitionen aus den Metropolen in die nachgeordneten Zentren in der Städte-Hierarchie ausgegangen werden, wie dies bisherige Ansätze zur räumlichen Ausbreitung von Gentrifizierungsprozessen im finanzdominierten Akkumulationsregime (vgl. Lees 2006; Fehlberg und Mießner 2015) - und in ähnlicher Weise auch ökonometrische Ansätze zum „Ripple-Effekt“, jedoch ohne Bezug auf Finanzialisierungsprozesse (Meen 1999; Kuethe und Pede 2011) - argumentieren.

Wir argumentieren in diesem Beitrag, dass hingegen die marxistische rent gap-Theorie von Neil Smith (2019 [1979]; 1996) geeignet ist, diese Prozesse immobilienwirtschaftlicher Aufwertungen in kleineren Städten zu erklären. Dieser Ansatz hat in den vergangenen Jahren in der internationalen (López-Morales 2010; Porter 2010; Slater 2017) wie in der deutschsprachigen Forschung (Belina 2011; Schipper 2013; Mießner 2019; sowie die Debatte in sub\urban. zeitschrift für kritische stadtforschung 2019) wieder verstärkt Aufmerksamkeit erfahren. Dieser Theorie folgend werden Kapitalinvestitionen an solchen Orten getätigt, wo die Lücke zwischen derzeitiger und potenziell erzielbarer Grundrente besonders groß ist. Dabei betont Smith, dass solche rent gaps nicht allein Ausdruck abstrakter ökonomischer Logiken sind, sondern auch lokal jeweils ermöglicht werden. 
Diesen Ansatz wollen wir im Folgenden zunächst in seinen Grundzügen vorstellen (Abschnitt 2). Darauf aufbauend führen wir in die Untersuchungsregion BerlinBrandenburg ein (Abschnitt 3) und erläutern das Forschungsdesign und die Fallstudienauswahl (Abschnitt 4). Daran anschließend zeigen wir anhand der Fallbeispiele Fürstenwalde (Spree; im Folgenden: Fürstenwalde) und Neuruppin die lokalen Bedingungskonstellationen auf, die im Fall von Fürstenwalde dazu führen, dass sich keine rent gap auftut, während sie sich in Neuruppin öffnet. Im Fazit diskutieren wir die Ergebnisse und unterstreichen, warum die rent gap-Theorie besonders fruchtbar zur Erklärung immobilienwirtschaftlicher Entwicklungen in mittelstädtischen Kontexten ist.

Mit der hier vorgeschlagenen Herangehensweise verorten wir uns innerhalb einer wirtschaftsgeographischen Diskussion, die seit einigen Jahren durch eine Vielfalt an Epistemologien, Theorien und Methodologien geprägt ist. Die Wirtschaftsgeographie zeichnet sich eher durch eine Heterodoxie als durch eine Orthodoxie aus (Sheppard 2018). Das heißt, sie verfolgt den Anspruch eines engagierten Pluralismus (Barnes und Sheppard 2010), der eine Verständigung zwischen den intellektuellen Inseln des Archipels der heterodoxen Wirtschaftsgeographie (Peck 2012, S. 128) einfordert. Eine dieser „Inseln“ bildet die neomarxistische Analyse der kapitalistischen Raumökonomie, welche Kapitalinvestitionen in städtische Wohnimmobilien als Teil der „gebauten Umwelt“ theoretisiert (Harvey 2006 [1982]).

\section{Rent gap-getriebene immobilien- wirtschaftliche Investitionen in kleineren Städten}

Kritische politisch-ökonomische Ansätze argumentieren, dass das Kapital an jene Orte fließt, wo es die besten Verwertungsbedingungen vorfindet (Smith 2010 [1984]), wobei die gebaute Umwelt eine bedeutende Rolle spielt (Harvey 2006 [1982]; Zeller 2003; Belina 2011).

Zur Erklärung, unter welchen Bedingungen Kapital in städtische Immobilienmärkte fließt und Aufwertungsprozesse in Gang setzt, hat sich die rent gap-Theorie von Neil Smith (2019 [1979]; vgl. auch 1996: 2006) etabliert. Der Begriff „rent“ wird für gewöhnlich als Miete oder Pacht übersetzt, in diesem Fall würde die rent gap also auf eine Mietlücke zwischen derzeit erzielter und potenziell erzielbarer Miete hinweisen. Der Neomarxist Smith nutzt den Begriff aber in einer anderen Konnotation, indem er sich auf die marxistische Grundrententheorie (Harvey 2006 [1982]) bezieht. Die Grundrente ist in dieser Theorietradition die „Geldsumme, die der Grundeigentümer jährlich aus der Verpachtung eines Stücks des Erdballs bezieht“ (Marx 2003 [1893], S. 636). Im Rahmen der kapitalistischen Konkurrenz verschiedener Kapitalien bzw. Investoren um die Nutzung des Bodens setzt sich dabei für gewöhnlich das Kapital bzw. der Investor durch, das/der in der Lage ist, die höchste Grundrente in der Form von Pacht oder Miete zu zahlen (Schipper 2013, S. 186). Die Grundrente bzw. der Wert des Bodens und der darauf befindlichen Gebäude wird dabei sowohl von den in der Umgebung befindlichen Flächen und darauf errichteten Strukturen als auch von dem Verkaufspreis der sich auf dem Boden vorhandenen Gebäude bestimmt (Ward und Aalbers 2016). Die Grundrente ist der neomarxistischen Theorie folgend nicht nur von Angebot und Nachfrage abhängig, sondern auch von anderen Faktoren, wie Lage oder Nutzungsart. Die rent gap ist demnach nicht als Mietlücke zu bezeichnen - letztere ist nur ein Faktor, der die rent gap ausmachen kann - sondern als die Lücke zwischen aktuell realisierter und potenzieller Grundrente (Schipper 2013). Ist diese Lücke ausreichend groß, so ist es für Investoren profitabel, in den entsprechenden Gebieten zu investieren. Dabei ist es aus Sicht des Kapitals irrelevant, ob es sich um einen Standort im Zentrum oder in der suburbanen Peripherie handelt. Entscheidend ist, dass die Renditeaussichten - in diesem Aufsatz im Sinne von Neil Smith verstanden als die ausreichend große Lücke zwischen derzeitig realisierter und potenziell erzielbarer Grundrente - gut sind und die Anlage am Standort als sicher genug gilt bzw. bei größerem Investitionsrisiko entsprechend höhere Renditen möglich sind. Dieser Logik folgend beginnt immobilienwirtschaftliche Aufwertung für gewöhnlich in innerstädtischen Räumen. Sind diese erst einmal aufgewertet, werden andere Orte, die geringere aber dennoch bedeutende Renditen versprechen, die nächsten Ziele der Kapitalinvestitionen (Smith 2019 [1979], S. 81).

Diese Argumentation wurde bisher hauptsächlich auf innerstädtische Aufwertungsprozesse in Großstädten angewandt und untersucht. Einzig die Arbeiten von Stefan Krätke (1991), Loretta Lees (2006), Paul Dutton (2005) und Fehlberg und Mießner (2015) haben diese rent gap-Theorie auf Prozesse entlang der Städte-Hierarchie übertragen, dabei aber wiederum ausschließlich Großstädte untersucht. Zudem unterstellen diese Ansätze ein „gentrifying down the urban hierarchy“ (Lees 2006), d.h. eine räumliche Ausbreitung von Aufwertungsprozessen auf nachgeordnete Zentren, sobald in den ranghöchsten Städten eine „Kapitalsättigung“ vorliege (Atkinson und Bridge 2005). Ähnlich argumentieren Ansätze der „mainstream econo- 
metrics“ (Meen 1999, S. 733), welche die wellenartigen räumlichen Ausbreitungseffekte von Schocks auf regionalen Wohnungsmärkten auf nahegelegene Teilmärkte mit dem Ripple-Effekt erklären (ebd.; Kuethe und Pede 2011). Demnach diffundieren Preisentwicklungen auf Immobilienmärkten etwa von urbanen Zentren ins Umland, indem bspw. aufgrund erschwinglicherer Preise in der Peripherie residentielle Migrationsströme von Haushalten mit höherer Kaufkraft dorthin ausgelöst werden oder Investoren Raumarbitrageeffekte in preisgünstigeren Nachbarregionen ausnutzen, wo sie Potentiale für höhere Preissteigerungen erkennen (Kuethe und Pede 2011, S. 564; Musil 2019, 149f.).

Gegenüber diesen theoretisch angenommenen Automatismen, welche in der rent gap-Literatur bislang anhand von Fallstudien sowie in der Literatur zum Ripple-Effekt mittels raumökonometrischer Modellverfahren quantitativ untersucht worden sind, unterstellt die rent gap-Theorie im Sinne von Neil Smith jedoch keine abstrakten Gesetzmäßigkeiten einer notwendigen räumlichen Ausbreitung von Aufwertungsprozessen. Im Sinne historisch-materialistischer Theoriebildung wird hier vielmehr betont, dass die rent gap gesellschaftlich unter verschiedenen historischen Bedingungen immer wieder neu (re-)produziert wird. Es sind demzufolge die konkreten lokalen Konstellationen aus angebots- und nachfrageseitigen Entwicklungen sowie stadtpolitischen Interventionen empirisch zu untersuchen, die eine Stadt $\mathrm{zu}$ (k)einem profitablen Investitionsstandort machen (Dutton 2005). Bspw. könnte sich das in Wohnimmobilien investierte Kapital ohne eine entsprechende zahlungskräftige Wohnungsnachfrage nicht verwerten. Die Betonung liegt hier auf zahlungskräftiger Nachfrage, weil ohne eine entsprechende finanzielle Ausstattung der Nachfragenden auch durch Wohnungsanbietende keine hohen Miet- und Immobilienpreise erzielt werden können. Z. B. sind Geringverdienende oder Studierende für gewöhnlich nur in der Lage, geringere Mieten zu zahlen. Dies hat zur Folge, dass sie entweder nur in Wohnungen mit geringerer Miete ziehen - oder eine ausreichend große Konkurrenz um Wohnraum vorausgesetzt - bereit sein müssen, in kleinere Wohnungen zu ziehen, weil die Eigentümerinnen und Eigentümer mit dieser Bevölkerungsgruppe nur hohe Renditen erwirtschaften können, wenn sie bspw. für kleine Wohnungen eine geringe Gesamtmiete, aber hohe Quadratmetermieten erzielen (vgl. Miessner 2021). Rent gaps können sich deshalb in Klein- und Mittelstädten auch nur dann auftun und kapitalisiert werden, wenn lokal eine ausreichend große zahlungskräftige Nachfrage vorhanden ist. Diese kann - muss aber nicht - durch verdrängungsinduzierte Wanderungsbewegungen aus den Metropolen in das Umland (Hierse et al. 2017) ausgelöst werden. Bisher fehlt jedoch ein empirischer und theoretischer Fokus auf lokale Ausbreitungsbedingungen für rent gaps in kleineren Städten.

\section{Immobilienmarktentwicklungen in Brandenburgs Zweiter Reihe}

Nach diesen Erläuterungen zu den Bedingungen rent gap-getriebener Investitionen in kleineren Städten richtet dieser Abschnitt den Fokus auf erste Anhaltspunkte für eine solche Entwicklung in Brandenburg. Die Veränderungen auf dem Brandenburger Immobilienmarkt stehen in engem Zusammenhang mit denen in der Hauptstadt Berlin. Diese war seit der Wiedervereinigung der wichtigste Wachstumsmotor der Region. Der damit verbundene Urbanisierungsprozess konzentrierte sich zunächst auf Berlin und das unmittelbare Umland. Die immobilienwirtschaftliche „Aufwertungsspirale“ (Döring und Ulbricht 2016) und Gentrifizierung begannen im Berliner Zentrum. Im Rahmen dessen wurden kaskadenförmige Umzugsprozesse in Gang gesetzt, die zur Folge haben, dass es zu residentieller Migration von innerstädtischer Bevölkerung an den Stadtrand und von Stadtrandbewohnerinnen und -bewohnern in den suburbanen Gürtel Berlins kommt (Förste und Bernt 2016; Hierse et al. 2017). Dieser Suburbanisierungsprozess erstreckt sich seit einiger Zeit auch auf die sogenannten „Städte der zweiten Reihe“ (Gemeinsame Landesplanungsabteilung Berlin-Brandenburg 2019, S. 69), die etwas entfernteren Mittelstädte Brandenburgs, die verkehrsinfrastrukturell gut angebunden sind und lange Zeit durch Bevölkerungsverluste geprägt waren (Gemeinsame Landesplanungsabteilung Berlin-Brandenburg 2018). Darüber hinaus finden in Brandenburg innerregionale Konzentrationsprozesse der Wirtschaftsund Beschäftigtenentwicklung statt (ebd.), von denen die Mittelstädte als „kleine Schwarmstädte“ (Simons et al. 2016) profitieren. Die neue prosperierende Entwicklung drückt sich dabei auch in gestiegenen Immobilien- und Wohnungsmieten in den Städten der zweiten Reihe aus (vgl. Oberer Gutachterausschuss für Grundstückswerte im Land Brandenburg 2018), sodass Immobilieninvestoren argumentieren, ,,[d]ie zweite Reihe rückt nach vorne“ (TAG Immobilien AG 2018, S. 13). Damit werden die Mittelstädte Brandenburgs mit guter verkehrsinfrastruktureller Anbindung an Berlin zunehmend attraktiv für Immobilieninvestitionen. Wie sich diese Tendenzen auf lokalen Immobilienmärkten konkret ausprägen, stellen wir im Folgenden für zwei solcher Mittelstädte dar. 


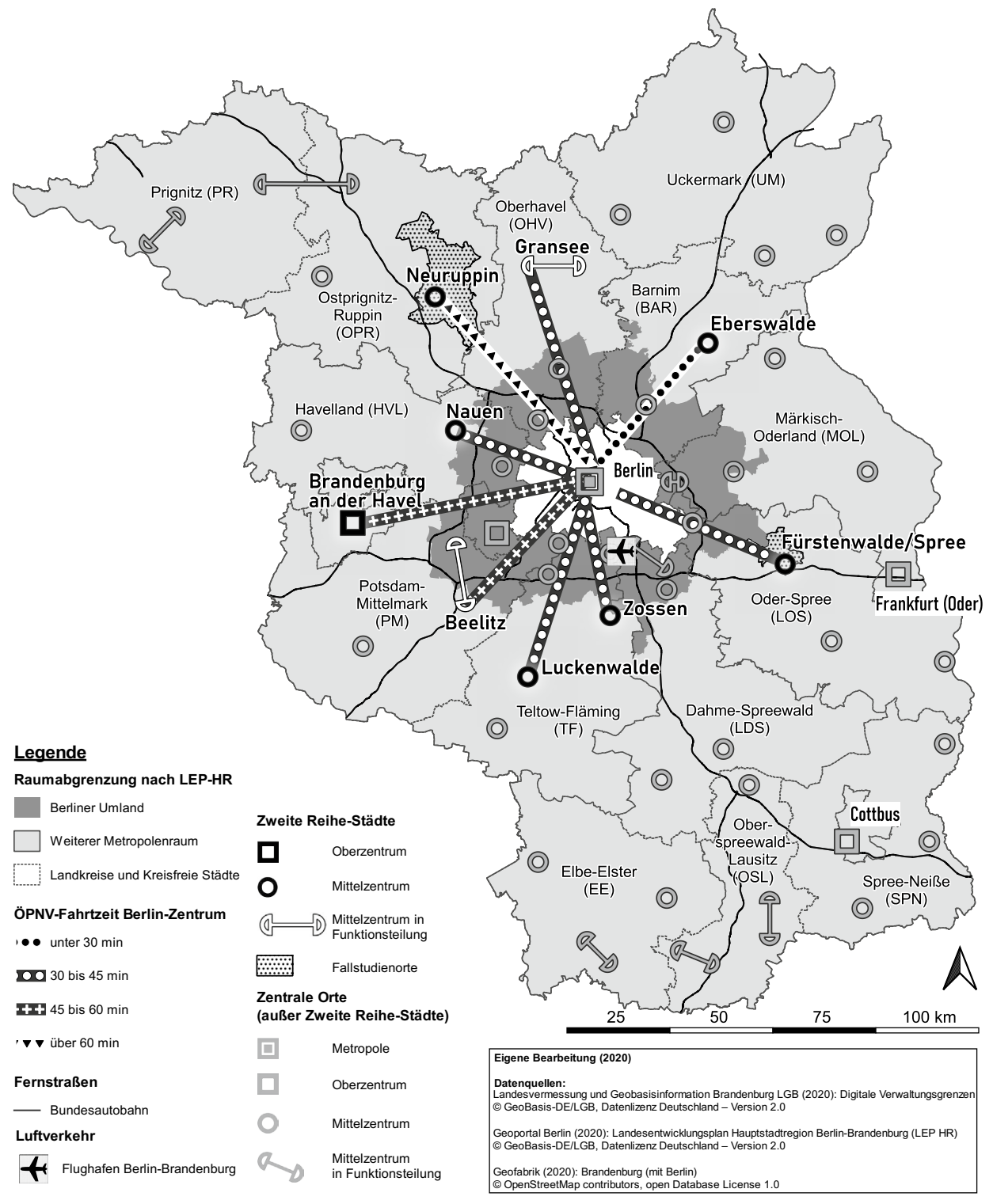

Abbildung 1: Lage und Erreichbarkeit der Zweite Reihe-Städte Brandenburgs

\section{Fallstudienauswahl und metho- disches Vorgehen}

Zur empirischen Untersuchung der räumlichen Ausbreitung immobilienwirtschaftlicher Aufwertung auf Mittelstädte in Brandenburg wurde ein qualitatives, komparatives Fallstudienvorgehen (vgl. Nijman 2007; Krehl und Weck 2020) gewählt. Dem historisch-materialistischen Ansatz folgend, dass rent gaps immer ein Resultat gesellschaftlicher räumlicher Praxis sind und somit die Ausbreitung des Anlagedrucks auf mittelstädtische Immobilienmärkte nicht per se angenommen werden kann, erfolgte eine Fallstudienauswahl nach dem Streuungsprinzip („most dissimilar cases“; Behnke et al. 2010, S. 204 ff.), um kontrastierend lokal-spezifische Ausbrei- tungsbedingungen für rent gaps in Mittelstädten herauszuarbeiten.

Im Rahmen der kriteriengeleiteten Auswahlstrategie für qualitative Stadtvergleiche (Sept 2018) wurden schrittweise zwei kontrastierende Fallstudienorte identifiziert: Da in anderen Studien bereits die Ausbreitung immobilienwirtschaftlicher Investitionen innerhalb einer eng abgegrenzten Metropolregion untersucht worden sind (Lees 2006; Porter 2010), wurden erstens bei der Auswahl der Fallstudien keine Städte berücksichtigt, die zum (unmittelbaren) Berliner Umland (Gemeinsame Landesplanungsabteilung Berlin-Brandenburg 2019) zählen (siehe Abbildung 1). Ausgehend vom Forschungsinteresse der Forschungsfrage wurde in einem zweiten Schritt auf Städte der zweiten Reihe fokussiert, dabei jedoch diejenigen Städte ausgeschlossen, die eine ÖPNV-Fahrtzeit von 


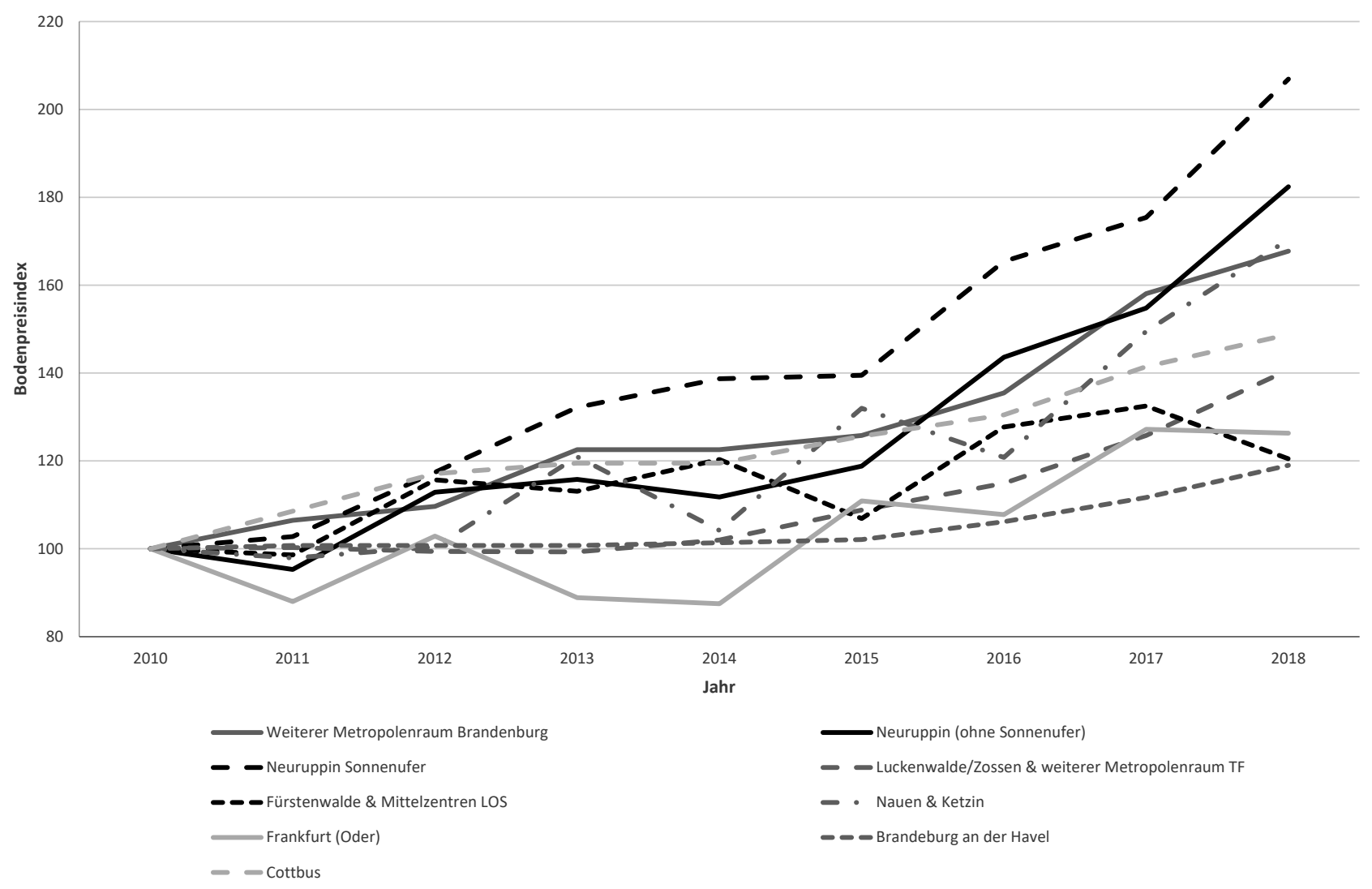

Abbildung 2: Entwicklung des Bodenpreisindex in Brandenburgs Städten der zweiten Reihe, nach Ausschluss von Städten mit weniger als 30 min. Fahrtzeit ins Zentrum Berlins und von Mittelzentren mit Funktionsteilung, und Oberzentren im weiteren Metropolenraum (Datenquellen: Oberer Gutachterausschuss für Grundstückswerte im Land Brandenburg 2018 sowie Gutachterausschüsse für Grundstückswerte in den Landkreisen Havelland 2019, Oder-Spree und in der Stadt Frankfurt (Oder) 2019, Ostprignitz-Ruppin 2019, Teltow-Fläming 2019, Stadt Brandenburg 2019, Cottbus 2019)

unter 30 min. zum Stadtzentrum Berlins ${ }^{1}$ aufweisen. Die ÖPNV-Fahrtzeit wurde als Auswahlkriterium herangezogen, um Orte auszuschließen, die ähnlich gut an das Berliner Zentrum angebunden sind wie die Berliner Umlandgemeinden. Außerdem wurde als Auswahlkriterium die Position in der Städte-Hierarchie des Zentrale-Orte-Systems in der Hauptstadtregion Berlin-Brandenburg herangezogen. Es wurden Ober- und Mittelzentren berücksichtigt und Mittelzentren mit Funktionsteilung ausgeschlossen. In einem dritten Schritt wurden Sekundärdaten der brandenburgischen Städte der zweiten Reihe zusammengetragen (siehe Tabelle 1). Ein besonderes Problem bei der Zusammenstellung der Sekundärdaten stellt die unzureichende Datenverfügbarkeit beim Vergleich von Mittelstädten dar, insbesondere auf kleinräumiger Ebene (Porsche et al. 2019, S. 52; Reimann et al. 2018). Auf dieser Datenbasis wurde das Sample schließlich kriteriengeleitet schritt-

1 Es wurden jeweils die minimalen Fahrtzeiten mit dem ÖPNV bis zum nächstgelegenen im Berliner Ringbahnbereich lokalisierten Fernbahnhof (Hauptbahnhof, Gesundbrunnen, Ostbahnhof, Südkreuz) erhoben. weise auf zwei kontrastierende Fallstudienorte reduziert. Dabei wurde der Entwicklung der Bodenpreisindizes für den Zeitraum 2010 bis 2018 für das Sample ein besonderes Gewicht beigemessen (Abbildung 2). Dies geschah, da steigende Bodenpreise auf sich öffnende rent gaps hinweisen, wie deutschsprachige Arbeiten zur Operationalisierung der Grundrente zeigen (Krätke 1991; Schipper 2013).

Die Mittelzentren Fürstenwalde und Neuruppin erweisen sich nach diesen Auswahlkriterien als die am stärksten kontrastierenden Fälle. Bei ähnlicher Bevölkerungszahl unterscheiden sich diese Städte der zweiten Reihe deutlich hinsichtlich ihrer ÖPNV-Fahrtzeit ins Berliner Stadtzentrum sowie der in diesem Beitrag im Zentrum stehenden Preisentwicklung am Grundstücksmarkt. Während die Bodenpreissteigerungen in Fürstenwalde trotz der schnellen Berlin-Verbindung von knapp über $30 \mathrm{~min}$. stagnieren, weist Neuruppin trotz einer langen Fahrtzeit von über einer Stunde mit $82 \%$ bzw. $107 \%$ die zweithöchsten Bodenpreissteigerungen unter den untersuchten Zweite Reihe-Städten auf (zentrale Daten sind für die beiden Fallstudienstädte in Tabelle 2 dargestellt). 
Tabelle 1: Fallstudienauswahl von Brandenburgs Zweite Reihe-Städten, nach Ausschluss von Städten mit weniger als 30 min. Fahrtzeit ins Zentrum Berlins und von Mittelzentren mit Funktionsteilung (Datenquellen: Gemeinsame Landesplanungsabteilung Berlin-Brandenburg 2019, S. 9, Deutsche Bahn 2020, Amt für Statistik Berlin-Brandenburg 2020, Gutachterausschüsse für Grundstückswerte in den Landkreisen Havelland 2019, Oder-Spree und in der Stadt Frankfurt 2019, Ostprignitz-Ruppin 2019, Teltow-Fläming 2019, in den Stadt Brandenburg 2019, Cottbus 2019).

\begin{tabular}{llllll}
\hline Ort & $\begin{array}{l}\text { ÖPNV-Fahrtzeit } \\
\text { nach Berlin- } \\
\text { Zentrum in } \\
\text { Minuten }\end{array}$ & $\begin{array}{l}\text { Funktion in Zentrale- } \\
\text { Orte-Hierarchie der } \\
\text { Hauptstadtregion }\end{array}$ & $\begin{array}{l}\text { Bevölkerungs- } \\
\text { zahl } \\
\mathbf{( 3 1 . 1 2 . 2 0 1 8 )}\end{array}$ & $\begin{array}{l}\text { Landkreis } \\
\text { Steigerung } \\
\text { Bodenpreisindex } \\
\mathbf{2 0 1 0 - 2 0 1 8}\end{array}$ \\
\hline Brandenburg an der Havel & 49 & Oberzentrum & 72.124 & Kreisfreie Stadt & $\begin{array}{l}19 \% \\
\text { Fürstenwalde (Spree) }\end{array}$ \\
Luckenwalde & 31 & Mittelzentrum & 31.941 & Oder-Spree & $20 \%$ \\
Nauen & 32 & Mittelzentrum & 20.522 & Teltow-Fläming & $41 \%$ \\
Neuruppin & 33 & Mittelzentrum & 17.967 & Havelland & $70 \%$ \\
Zossen & 64 & Mittelzentrum & 30.846 & Ostprignitz-Ruppin & $82 \%$ \\
\hline
\end{tabular}

Tabelle 2: Übersicht zentraler Daten der Fallstudienorte (Datenquellen: B.B.S.M. 2018, Empirica-Systeme Marktdatenbank 2020, Gutachterausschuss für Grundstückswerte im Landkreis Oder-Spree und in der Stadt Frankfurt (Oder) 2019, Gutachterausschuss für Grundstückswerte im Landkreis Ostprignitz-Ruppin 2019, Institut für Wohnen und Stadtentwicklung 2019, Perrot 2019, Stadt Fürstenwalde 2019b)

\begin{tabular}{lrr}
\hline Indikator & Fürstenwalde & Neuruppin \\
\hline Fahrtzeit ins Berliner Stadtzentrum mit ÖPNV & $31 \mathrm{~min}$. & $64 \mathrm{~min}$. \\
Bevölkerungszahl (2018) & 33.200 & 31.600 \\
Bevölkerungsentwicklung 2012-2018 & $3,6 \%$ & $7,7 \%$ \\
Leerstand & $4,9 \%(2016)$ & $3,0 \%(2019)$ \\
Angebotsmietpreis (2019) & $6,96 € / \mathrm{m}^{2}$ & $7,60 € / \mathrm{m}^{2}$ \\
Entwicklung der Angebotsmieten 2012-2019 & $26,8 \%$ & $47,9 \%$ \\
Bodenpreisindexentwicklung (2010-2018) & $+25,4$ & $+82,4^{\star}$ \\
\hline
\end{tabular}

* ohne Sonnenufer

Nach dieser Auswahl der Fallstudienorte wurden die Entwicklungen in den Fallstädten erforscht. Dazu wurden an beiden Orten insgesamt 19 (8 in Fürstenwalde, 11 in Neuruppin) qualitative, problemzentrierte Interviews (Lamnek 2010, 332ff.) mit Expertinnen und Experten der Stadtplanung, der Stadterneuerung und der Gutachterausschüsse für Grundstückswerte sowie mit den Immobilienabteilungen von Banken, mit Wohnungsbauträgern und Investoren geführt und anschließend transkribiert. Mittels eines Mix aus offenem und thematischem Kodieren (Mattissek et al. 2013, $201 \mathrm{ff}$.) wurde das Interviewmaterial anschließend iterativ verdichtet, wodurch Problemfelder und Wahrnehmungen der lokalen Akteure gemäß des rekonstruktiven Paradigmas (Bohnsack 2014) herausgearbeitet wurden. Dieses qualitative Vorgehen ist aus zwei Gründen erforderlich. Erstens ist eine rein deskriptive Betrachtung von Bodenpreisindizes und Bodenrichtwerten der Gutachterausschüsse allein nicht ausreichend, um einen Anstieg der realen Grundrente zu erkennen, da „die Bodenrichtwerte spekulative Entwicklungen immer nur zeitversetzt widerspiegeln können, sie also erst erfassen, nachdem sich Erwartungen auf eine zukünftig höhere potenzielle Grundrente bereits in steigenden Bodenpreisen niedergeschlagen haben“" (Schipper 2013, S. 189 f.) und somit Frühphasen kapitalgetriebener immobilienwirtschaftlicher Aufwertung nur schwer erkennbar sind. Zweitens begründet sich ein qualitatives Vorgehen in der spekulativen Form der Grundrentensteigerung, die untrennbar von der gesellschaftlichen Praxis ist. Der hier verfolgte historisch-materialistische Ansatz, der rent gaps als Produkt des Zusammenspiels zwischen allgemeinen kapitalistischen Logiken und lokal-spezifischen Akteurskonstellationen begreift, muss folglich untersuchen, wie rent gaps konkret auf lokaler Ebene hervorgebracht werden. Somit sind Handlungen und Rationalisierungen der an lokalen Grundstücksmarktentwicklungen beteiligten Akteure (Holm 2011) wichtiger Bestandteil qualitativer rent gap-Forschung. Diese Akteure werden hierbei verstanden als „Charaktermasken“ (Marx 1962 [1867], S.100), also als „Abstraktionen, die so agieren, wie es ihre Position innerhalb der eingerichteten Gesetzmäßigkeiten der Kapitalzirkulation von ihnen verlangt, und 
die so agieren können, wie es eben jene Position ermöglicht“"(Belina 2017, S. 31). Demnach werden Eigentümerinnen und Eigentümer oder Investoren eine Steigerung auf eine höhere potenzielle Grundrente (spekulativ) durchsetzen, sofern sie die Bedingungen für deren Kapitalisierung zukünftig am lokalen Immobilienmarkt als gegeben wahrnehmen. Diese Wahrnehmungsmuster der Akteure und das Nachvollziehen lokal-spezifischer Entwicklungen können nur mittels qualitativer, rekonstruktiver Sozialforschung erhoben werden. Drittens folgt das Vorgehen damit den Erkenntnissen quantitativ ausgerichteter Gentrifizierungsforschung, die eine Ergänzung um qualitative Interviews als zielführende Vertiefung bezeichnet (Beran und Nuissl 2019, S. 203), sowie Forschungen zu ländlicher Gentrifizierung (z. B. Sakarya und Başaran Uysal 2018), die zeigen, dass solche Prozesse abseits der Großstädte am besten mit einem Methodenmix erforscht werden können.

\section{Bedingungen für Grundrenten- steigerungen in Mittelstädten: Fallstudien in Fürstenwalde und Neuruppin}

Im Folgenden wollen wir die Prozesse immobilienwirtschaftlicher Aufwertung im Spannungsfeld zwischen der Durchsetzung allgemeiner kapitalistischer Logiken und den lokalen Entwicklungen am Beispiel zweier Mittelstädte in Brandenburg untersuchen. Aufbauend auf die rent gap-Theorie erforschen wir dabei insbesondere, ob es Hinweise gibt, dass sich in diesen brandenburgischen Mittelstädten eine Renditelücke auftut. Dabei zeigen wir, dass in Fürstenwalde trotz neuer Dynamiken am Grundstücksmarkt nur schwache Grundrentensteigerungen vollzogen werden. In der zweiten Fallstadt Neuruppin sind demgegenüber bereits rent gap-Steigerungen nachweisbar.

\subsection{Fürstenwalde: Lokal begrenzte Angebots- und Nachfrageausweitung}

Im Mittelzentrum Fürstenwalde im Landkreis Oder-Spree (LOS) südöstlich von Berlin deuten die lokalen Immobilienmarktentwicklungen bislang auf keine Öffnung einer rent gap hin, wie wir in diesem Abschnitt zeigen. Dabei bewirbt sich die Stadt als attraktiver Wohnstandort für Pendlerinnen und Pendler (Stadt Fürstenwalde 2019a), da der Berliner Ostbahnhof in 31 min. und der Hauptbahn- hof in $43 \mathrm{~min}$. Fahrtzeit mit dem halbstündlich getakteten Schienenverkehr erreichbar sind. Aufgrund dieser Lagegunst und einer seit 2012 steigenden Bevölkerungszahl ist Fürstenwalde als Stadt der zweiten Reihe ein landesplanerisch begünstigter Standort für neue Siedlungsentwicklungen. Folglich geht die Planung von einem leichten zukünftigen Wachstum aus (GRUPPE PLANWERK 2018, S. 29), da aufgrund der Entwicklungen des Berliner Immobilienmarktes „der Druck auch auf die verfügbaren Wohnbauflächen in Fürstenwalde zunehmen“ (ebd., 13) werde. Allerdings spiegeln sich diese unterstellten Wachstumserwartungen bislang nur bedingt in den Boden- und Mietpreisentwicklungen wider. Die Bodenpreise stiegen zwischen 2010 und 2018 um rund 25 \%, was im Vergleich mit anderen Städten der zweiten Reihe im weiteren Metropolenraum niedrig ist (vgl. Abbildung 1). Auch die Steigerung der Angebotsmieten um 26,9\% von durchschnittlich $5,49 € / \mathrm{m}^{2}$ auf $6,97 € / \mathrm{m}^{2}$ im Zeitraum 2012 bis 2019 fällt deutlich schwächer aus als im zweiten Fallstudienort Neuruppin (vgl. Abbildung 3). Dieser Abschnitt beleuchtet im Folgenden die Ursachen für das Ausbleiben größerer Grundrentensteigerungen und somit der nicht erkennbaren Öffnung einer rent gap in Fürstenwalde.

\subsubsection{Nachfrageseitige Entwicklungen}

In Fürstenwalde lässt sich nur eine leichte Steigerung der Wohnungs- und Anlagenachfrage durch Mieterinnen und Mieter, Käuferinnen und Käufer sowie Investoren nachweisen. Erstens hat das leichte, durch einen positiven Wanderungssaldo bedingte, Bevölkerungswachstum von 3,6 \% zwischen 2012 und 2018 auf etwa 33.200 Einwohnerinnen und Einwohner (Stadt Fürstenwalde 2019b) keinen erheblichen Druck auf dem lokalen Wohnungsmarkt ausgelöst. Dieses Wachstum ist weniger auf Zuwanderungen aus der Bundeshauptstadt, sondern hauptsächlich auf Zuzüge von Geflüchteten zurückführbar (B.B.S.M. 2018, S. 12). Ein kleinerer Teil der Wanderungsgewinne ergibt sich durch Familienzuzüge aus dem Fürstenwalder Umland sowie aus Berlin (ebd., S. 9). Auch die Zuzüge aus Berlin haben bislang keinen nachfrageinduzierten Wachstumsdruck ausgelöst (I 12). Es fehle Fürstenwalde im Vergleich zu anderen Zweite Reihe-Städten in Brandenburg an Attraktivität, also einem Angebot weicher Standortfaktoren wie etwa Hochschulen oder baulich attraktiven Innenstädten (ebd.), was die fehlende Nachfrage aus Berlin nach Wohnraum in Fürstenwalde bedinge.

Zweitens wirken sich diese Zuwanderungsmuster auf die Zahlungsbereitschaft der Nachfragenden auf dem Mietwohnungs- und Grundstücksmarkt aus. In Fürsten- 


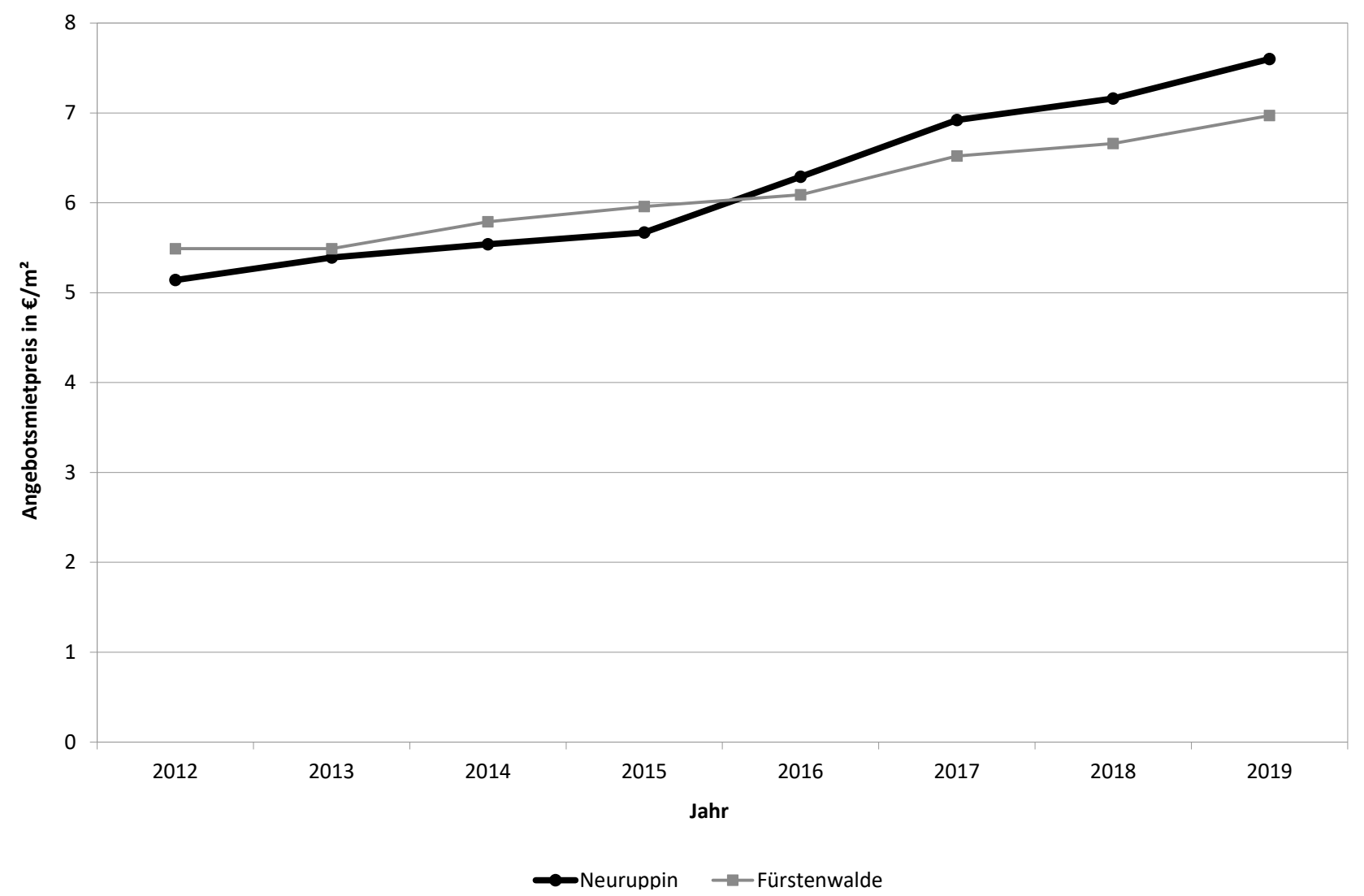

Abbildung 3: Entwicklung der Angebotsmietpreise in Fürstenwalde und Neuruppin 2012-2019.

(Datenquelle: Empirica-Systeme Marktdatenbank 2020).

walde lebende Geflüchtete fragen aufgrund der prekären Lebenssituation mit einer geringen Kaufkraft nur Wohnraum im preisgünstigen Mietsegment nach (I 12, 19). Auch die seit Mitte der 2010er Jahre deutlich gestiegene Nachfrage nach baureifem Land zur Wohnbebauung durch lokal ansässige bzw. aus dem Umland zugewanderte private Haushalte $(\mathrm{I} 15,17)$ hat bislang zu keinen starken Grundrentensteigerungen auf dem Fürstenwalder Grundstücksmarkt geführt, da bei Veräußerungen Kaufpreise von weniger als $100 € / \mathrm{m}^{2}$ für Wohnbauland aufgerufen wurden (I 12, 17, 18). Damit liegt das Preisniveau deutlich unter dem in Gemeinden des Berliner Umlandes (I 15). Aufgrund des Überhangs lokal ansässiger Käuferinnen und Käufer konnten angesichts des geringen Kaufkraftniveaus (I 13) - in Fürstenwalde lag dieses 2019 bei lediglich $84 \%$ des Bundesdurchschnitts (eigene Berechnungen nach Acxiom 2019) - nur relativ geringe Kaufpreise gezahlt werden. Allerdings erwarten die lokalen Akteure in Zukunft eine steigende Nachfrage nach höherpreisigem Wohnraum in Fürstenwalde (I 14, 17). Als Gründe werden die räumliche Nähe und gute Erreichbarkeit des 2020 in Betrieb gegangenen Flughafens BER (I 13, 14, 17) sowie der 2021 eröffnenden Tesla Giga Factory in der Nachbar- gemeinde Grünheide in 50 bzw. 20 Kilometer Entfernung genannt. Deren Beschäftigungsimpulse am regionalen Arbeitsmarkt würden künftig eine höhere Nachfrage zahlungskräftiger Interessentinnen und Interessenten nach privaten Einfamilienhäusern stimulieren.

Drittens verhindert die Art und Kapitalstärke der auf dem Fürstenwalder Wohnimmobilienmarkt aktiven Investoren rent gap-Steigerungen. Ein Ausweichen institutioneller Investoren von A-Standorten ins Umland durch Wohnbauentwicklungen oder den Erwerb von Mietwohnungsbeständen in Fürstenwalde ist nicht erkennbar. „[F]ür institutionelle, ausländische Investoren ist Fürstenwalde irgendwie auf dem Mond“ (I 13). Diese seien in Brandenburg nur an Projektentwicklungen mit mehreren tausend Wohneinheiten wie etwa Bernau Teufelspfuhl interessiert $(\mathrm{I} 12,16)$. Trotz bestehender Wohnbauflächenpotentiale (GRUPPE PLANWERK 2018, S. 42) ist die Größe derzeit entwickelbarer Wohnbauprojekte in Fürstenwalde irrelevant für kapitalstarke, überregionale Investoren: „Also die großen [Investoren] sind zurückhaltend [...], deutschlandweit oder gar global sind wir da nicht auf dem Schirm [...]. Da gibt's offensichtlich noch andere attraktive Flächen, um die sie sich eher kümmern“ (I 12). 
Als Grund für das Fehlen überregionaler Investoren wird vor allem das schlechte Image Ostbrandenburgs herangezogen: „In den Köpfen ist Fürstenwalde [...] noch immer irgendwo bei Warschau. [...] Man kennt [in Berlin] den Scharmützelsee oder Bad Saarow. Fürstenwalde nicht und dann, wenn Sie Fürstenwalde ins Spiel bringen, werden Sie ganz schnell zu Eberswalde, Finsterwalde, Freienwalde“ (I 13).

Zudem zeigen diese Investoren bisher kein Interesse an Immobilienentwicklungen in Fürstenwalde, da sich aufgrund ausbleibender Zuzüge zahlungskräftiger Nachfragender die Zahlungsbereitschaft der lokal ansässigen Nachfragenden für höhere Mieten und Kaufpreise nicht erhöht habe (I 12, 13).

\subsubsection{Angebotsseitige Entwicklungen}

Angebotsseitig verhindern erstens die Angebotsüberschüsse am städtischen Mietwohnungsmarkt Grundrentensteigerungen in Fürstenwalde. Trotz einer mehrjährigen Phase des Rückbaus in Beständen des Geschosswohnungsbaus aus DDR-Zeiten lag der Leerstand im Jahr 2016 noch bei 4,9 \% (B.B.S.M. 2018, S. 7) und damit über der Fluktuationsreserve von $3 \%$. Um der 2015 kurzfristig gestiegenen Nachfrage nach preisgünstigem Wohnraum nachzukommen, konnten zudem stillgelegte Etagen in Beständen des kommunalen Wohnungsunternehmens schnell zur Vermietung reaktiviert werden (I 13).

Zweitens werden die in Fürstenwalde nur geringen Grundrentensteigerungen von lokalen Kleininvestoren mit guter Kenntnis des regionalen Wohnungsmarktes vorangetrieben. Für diese sei der Umfang planerisch realisierbarer Wohneinheiten im niedrigen zweistelligen Bereich in Fürstenwalde attraktiv und umsetzbar (I 12, 17, 18). Hierbei werden nicht ausschließlich Einfamilienhäuser entwickelt, sondern neuerdings auch höherpreisige, mehrgeschossige Eigentumswohnungen, welche am Fürstenwalder Wohnungsmarkt ein neues Segment bilden. Mit diesen einzelnen innerstädtischen Objekten wollen regionale Investoren testen, ob höherpreisige Eigentumswohnungen in Fürstenwalde marktgängig sind und bewerben diese auch gezielt in Berlin oder bei Gästen im benachbarten Kurort Bad Saarow (I 13, 14). Regionale Investoren spekulieren daher auf einen künftig wachsenden Fürstenwalder Wohnungsmarkt, insbesondere durch Zuzüge einer zahlungskräftigeren Nachfrage aus Berlin, wobei sich diese Erwartungen bislang nicht auf die Entwicklung von Boden- und Mietpreisen auswirken.

Besonders bedeutsam für die jüngere Wohnraumentwicklung ist drittens die Kommune, die als größte Flächen- eigentümerin auftritt und sowohl das Flächenangebot als auch den Grad der Grundrentensteigerung in besonderem Maße steuert. Die Stadt war bis 1994 Militär- sowie zu DDRZeiten ein Industriestandort. Nach Abzug des Militärs und aufgrund der Deindustrialisierung nach 1990 verfügt die Stadt Fürstenwalde über zahlreiche innenstadtnahe Konversionsflächen, welche erst seit Kurzem auf steigendes Interesse von lokalen Immobilienentwicklerinnen und -entwicklern und Privathaushalten stoßen (vgl. Kap. 5.1.1) und auf denen landesplanerisch eine weitere Siedlungsentwicklung in der zweiten Reihe befürwortet wird (Gemeinsame Landesplanungsabteilung Berlin-Brandenburg 2019, S. 69). Diese Flächen werden durch die städtischen Liegenschaften zur Bebauung erschlossen und über Ausschreibungen privatisiert (I 17, 18).

Obwohl diese kommunalen Baugrundstücke - aufgrund fehlender Ausschreibungsbedingungen zur preisgebundenen Wohnraumförderung und zur Entlastung des kommunalen Haushaltes - nach dem Höchstgebotsverfahren veräußert werden, geht die Stadt bei diesen Grundstücksveräußerungen nicht grundrentenmaximierend vor: Die Fürstenwalder Stadtentwicklungspolitik und die jüngeren Grundstücksprivatisierungen sind auf lokal ansässige Mittelschichten ausgerichtet (I 12, 16). Um den großflächigen Ankauf durch private Großinvestoren zu verhindern, wurde bei der Privatisierung der ersten 36 Baugrundstücke im Gebiet ,Ketschendorfer Feldmark ${ }^{`}$ zwischen 2016 und 2018 auf eine überregionale Bewerbung der Grundstücke bewusst verzichtet und die Zahl der Gebote beschränkt, um die Flächen zum Einfamilienhausbau möglichst an ortsansässige Käuferinnen und Käufer zu veräußern, was auch gelang (I 17, 18). Das Mindestgebot für diese Grundstücke orientierte sich am ortsüblichen Bodenrichtwert, der in dieser Lage bei etwa $75 € / \mathrm{m}^{2}$ lag (I 18). Durch eine solche Privatisierungspolitik sorgt die Stadt für eine gedämpfte Preisentwicklung im Ein- und Zwei-Familienhausneubau.

Zusammengefasst lässt das Zusammenspiel von Angebot und Nachfrage in Fürstenwalde bisher keine rent gap-Steigerungen zu. Erstens liegt dies am Verharren der Zahlungsbereitschaft der Nachfragenden auf niedrigem Niveau aufgrund der unterdurchschnittlichen lokalen Kaufkraft und fehlender zahlungskräftiger Zuwandernden sowie einer bisher ausbleibenden Nachfrage überregionaler Investoren. Zweitens wurde einer wachsenden Nachfrage mit einer Ausweitung des Angebots kurzfristig durch die Reaktivierung von Leerstand und mittelfristig durch die Privatisierung kommunaler Flächen am Grundstücksmarkt begegnet, wobei die Stadt als Flächeneigentümerin auf die Erzielung niedriger Grundstückspreise für lokale Käuferinnen und Käufer durch ihren Vergabemechanis- 
mus hinwirkte. Ein fehlender Zustrom von überschüssigem Kapital durch größere, überregionale Investoren und ein damit verbundener Zwang zur Durchsetzung höherer Renditen am Immobilienmarkt sind in Fürstenwalde folglich nicht gegeben, da Wohnbauflächen und Wohnungsbestände trotz vorhandener Entwicklungspotentiale nicht den Renditeerwartungen dieser Akteurstypen entsprechen. Stattdessen wird die Angebotsausweitung am Immobilienmarkt durch lokale Investoren entwickelt, die sich wiederum am Kaufkraftniveau einer lokalen Nachfrage orientieren.

\subsection{Neuruppin: Aufwertung und erste Investitionen überregionaler Kapital- geber}

Etwas anders als in Fürstenwalde stellt sich die Situation im rund 80 Kilometer nordwestlich von Berlin gelegenen Neuruppin dar, der Kreisstadt des Landkreises OstprignitzRuppin (OPR). Obwohl die Stadt im Vergleich zu anderen Städten in der zweiten Reihe mit einer langen Bahnfahrtzeit von $1 \mathrm{~h} 4$ min. nach Berlin Gesundbrunnen und $1 \mathrm{~h}$ 19 min. zum Berliner Hauptbahnhof benachteiligt ist, sind hier die Bodenpreise überdurchschnittlich stark angestiegen (vgl. Abbildung 2). Die Preise sind in Neuruppin seit 2010 um mehr als $80 \%$ angestiegen. Noch deutlicher fiel die Entwicklung im neu entstandenen Quartier Sonnenufer aus, in dem sich die Preise im gleichen Zeitraum mehr als verdoppelten. Ausgehend von einem höheren Ausgangsniveau sind die Bodenpreise in der Stadt Neuruppin nach 2014 auch wesentlich stärker gestiegen als in den übrigen Städten des Landkreises OPR (Gutachterausschuss für Grundstückswerte im Landkreis OstprignitzRuppin 2019). Der Grundrententheorie folgend deutet diese Entwicklung darauf hin, dass sich in der Stadt eine rent gap auftut. Dafür spricht, dass auch die Angebotsmietpreise von 5,14 €/m² im Jahr 2012 auf 7,60 €/m² 2019 um über $45 \%$ und damit sehr stark gestiegen sind (vgl. Abbildung 3). Die Ursachen dieser Entwicklungen sind Gegenstand dieses Abschnittes.

\subsubsection{Nachfrageseitige Entwicklungen}

Eine erste nachfrageseitige Ursache für die sich auftuende Renditelücke in der Stadt ist die erhöhte Wohnungsnachfrage. Die Bevölkerung ist seit 2012 um mehr als 800 Personen bzw. 7,7 \% angewachsen auf mittlerweile knapp 31.600 Einwohnerinnen und Einwohner (eigene Berechnungen nach Perrot 2019). Entsprechend wird in nahezu allen Interviews eine gestiegene Wohnungsnachfrage für Neuruppin konstatiert. Diese zeige sich in einem Bevölkerungszuzug (I 2, 6), einer vermehrten Nachfrage von Berlinerinnen und Berlinern (I 2, 6, 7) sowie einer gestiegenen Nachfrage aus dem Neuruppiner Umland (I 2, 7, 8, 9). Insbesondere Familien und ältere Menschen würden in die Stadt ziehen, weil hier alle notwendigen Güter des alltäglichen Bedarfs sowie Kindergärten, Schulen und Gesundheitsvorsorgeeinrichtungen gut und überwiegend fußläufig erreichbar seien (I 2, 3, 4). Diese Einschätzung wird durch die jüngste Wohnungsmarktstudie gestützt, die zeigt, dass die Stadt seit dem Jahr 2015 einen Wanderungsüberschuss von 1.700 Personen verzeichnet, wovon ein Drittel aus dem Landkreis OPR und weitere zehn Prozent aus Berlin zuziehen (Institut für Wohnen und Stadtentwicklung 2019, S. 36-38).

Neben der gestiegenen quantitativen Zahl der Nachfragenden ist zweitens zu konstatieren, dass zahlungskräftigere Klientel Wohnungen in der Stadt nachfragen. Dies hat zwei Ursachen. Zum einen käme rund die Hälfte der Interessentinnen und Interessenten für Wohneigentum aus Berlin (I1). Diese Nachfragenden seien aus Berlin deutlich höhere Immobilien- und Mietpreise gewöhnt, weshalb die in Neuruppin zu zahlenden Preise als wesentlich günstiger wahrgenommen werden (I 2, 3). Während es mit dem verfügbaren Einkommen auf dem Berliner Wohnungsmarkt schwierig werden würde, eine passende Wohnung bzw. Immobilie zu finanzieren, weisen diese Haushalte in Neuruppin eine vergleichsweise große Zahlungskraft auf und können auch für die Stadt hohe Immobilien- und Mietpreise zahlen (I 6). Zum anderen wird die zahlungskräftige Nachfrage insbesondere durch die ortsansässigen Behörden (Stadt- und Kreisverwaltung, Landgericht, Polizeidirektion) und die Ruppiner Klinken generiert (vgl. Ernst Basler + Partner 2013, 31-32). Die dort angestellten Mitarbeiterinnen und Mitarbeiter, Ärztinnen und Ärzte sowie Hochschulprofessorinnen und -professoren sind in der Lage und auch bereit, höhere Miet- wie auch Wohneigentumspreise zu zahlen (I 1, 2, 3, 4).

Drittens erläutern die Interviewten, dass mehr externe, insbesondere Berliner Investoren auf den Neuruppiner Markt drängen (z. B. I 2, 4, 9), welche aktiv werden, sobald Häuser mit mehr als sechs Wohneinheiten zum Verkauf stehen (I 4):

„Es gibt zum Beispiel Investoren, die sich vorher [...] auf den Berliner Markt konzentriert haben. Wo aber nicht mehr ganz so viel zu verdienen ist, renditetechnisch. [...W]enn man da [in Berlin] jetzt ein Mehrfamilienhaus kauft, kriegt man da ja kaum noch Rendite. [...] Und da gucken natürlich auch viele Investoren [...] was gibt es da noch so im Umland? [...] Gerade Neuruppin [...] ist da auch in den Fokus von vielen Investoren gelangt. Also es 
gibt Leute, die kaufen hier auch, im Altstadtbereich Mehrfamilienhäuser und vermieten die dann, weil sie eine bessere Rendite als in Berlin erzielen“ (I 1).

\subsubsection{Angebotsseitige Entwicklungen}

Angebotsseitig lässt sich erstens feststellen, dass der Angebotsüberschuss in den vergangenen Jahren abgebaut wurde. So ist die Leerstandsquote seit 2011 von 5,6 \% auf aktuell drei Prozent gesunken (Institut für Wohnen und Stadtentwicklung 2019) und hat damit die Fluktuationsreserve erreicht. Dies dürfte zweitens auch darin begründet sein, dass die Stadt angesichts eines langfristig erwarteten Bevölkerungsrückgangs bemüht ist, kein „übermäßiges Flächenangebot zu produzieren“ (Fontanestadt Neuruppin 2017) und somit auf Innenentwicklung und nicht auf Flächenentwicklungen auf der „grünen Wiese“ (I 9) setzt.

Drittens werden in Neuruppin durch lokale Projektentwickler Angebote geschaffen, die sich an private Finanzanleger richten. Insbesondere die Prima-Gruppe hat dieses Geschäftsmodell in Neuruppin in der jüngsten Vergangenheit vorangetrieben. Das zurzeit prestigeträchtigste Projekt der Prima-Gruppe in Neuruppin ist die Entwicklung der „Seetor Residenz“. Auf dem direkt am Ruppiner See gelegenen Grundstück sind bisher $5.560 \mathrm{~m}^{2}$ Wohnfläche entstanden bzw. ist die Bebauung mit weiteren $16.000 \mathrm{~m}^{2}$ Bruttogeschossfläche geplant (Prima Gruppe 2019). Zielgruppe des Projektes sind dabei weniger Personen aus Neuruppin als überregionale private Finanzanleger. Dafür wurde eigens ein „schönes Hochglanzprospekt“ (I 3) erstellt, in dem auch die möglichen Eigenkapitalrenditen von 3,5\% bis 8,2 \% für den Kauf der unterschiedlichen Wohnungen erläutert werden. Die Verkaufspreise der bisher veräußerten Wohnungen bewegten sich zwischen $2.790 € / \mathrm{m}^{2}$ und $4.200 € / \mathrm{m}^{2}$ (eigene Berechnungen nach Seetor Residenz Neuruppin 2015) und liegen damit weit über dem in Neuruppin üblichen Preis (I 3). Ebenso sind die in dem Prospekt veranschlagten Nettokaltmieten von $12,00 € / \mathrm{m}^{2}$ bis $14,50 € / \mathrm{m}^{2}$ im Neuruppiner Vergleich deutlich überdurchschnittlich. Trotz dieser für die Stadt hohen Preise hat dieses Projekt „deutschlandweit richtig Erfolg gehabt" und die Wohnungen des ersten Bauabschnittes konnten als Eigentumswohnungen fast ausnahmslos verkauft werden (I 3). Diese wurden hauptsächlich von privaten Anlegerinnen und Anlegern aus dem süddeutschen Raum als Anlageobjekte erworben (ebd.), denen die versprochenen Renditen angesichts ihrer dortigen Erfahrungen mit angespannten Immobilienmärkten realistisch erschienen, die Wohnungen als Anlageobjekte kauften und nun an ein zahlungskräftiges Publikum oder als gehobene Ferienwohnungen vermieten. Selbst lokale Marktkennerinnen und -kenner sind von dem Erfolg des Projektes überrascht, wie die Aussage eines Immobilienmaklers deutlich macht: „Erstaunlich für Neuruppin in diesem Sektor, muss man unumwunden zugeben“ (I 3).

In diesem Zusammenspiel aus nachfrageseitigen und angebotsseitigen Faktoren wird die Durchsetzung höherer Renditen möglich und damit eine rent gap in Neuruppin geöffnet: So bietet die erhöhte Wohnungsnachfrage und damit einhergehende verstärkte Konkurrenz bei gleichzeitig nur geringer Flächenausweisung ein Umfeld, das Investoren die Durchsetzung höherer Miet- und Kaufpreise ermöglicht. Die in der Stadt zugleich vorherrschende gute wirtschaftliche Situation (Ernst Basler + Partner 2013) bedingt eine vergleichsweise zahlungskräftige Nachfrage. Diese wird durch eine überregionale Nachfrage von privaten Finanzinvestoren ergänzt, die durch eine aufwändige Vermarktung durch lokale Projektentwickler in die Stadt geleitet wird. Im Rahmen dieser Konstellation testen einige Investoren sowie Vermieterinnen und Vermieter, wie weit sie die Mieten steigern können, wie im Interview deutlich wurde (I 11). Das Beispiel Neuruppin macht aber auch die Bedeutung weiterer Forschung zur Rolle lokaler Stakeholder mit einer Scharnierfunktion zu überregionalen privaten Finanzinvestoren deutlich. Solche Immobilienentwickler produzieren überhaupt erst gute Immobilienlagen und somit potentielle rent gaps. Sie sind wichtig, um Kapital in mittelstädtische Immobilienmärkte zu kanalisieren.

\section{Fazit}

Die Entwicklungen in Fürstenwalde und Neuruppin zeigen, dass einige Mittelstädte jenseits der Metropolen durch neue Dynamiken immobilienwirtschaftlicher Aufwertung gekennzeichnet sind. Dabei ist die Erklärung solcher Prozesse durch die wirtschaftsgeographische Finanzialisierungsdiskussion - aber auch durch ökonometrische Ansätze - bisher nicht ausreichend. Einerseits ist bisher keine ausreichende Markttransparenz mittelstädtischer Immobilienmärkte hergestellt, die letztere mit großstädtischen Märkten vergleichbar machen würde. Andererseits macht unsere Studie deutlich, dass sich Immobilieninvestitionen nicht gesetzmäßig und wellenförmig vom Zentrum her ausbreiten. Gerade Fürstenwalde wäre hinsichtlich seiner Berlin-nahen Lage gegenüber Neuruppin prädestiniert für steigende Immobilieninvestitionen und damit einhergehenden rent gap-Steigerungen. Unsere Forschungsergebnisse zeigen aber, dass sich das 
tatsächliche Investitionsgeschehen genau entgegengesetzt verhält.

Diese Entwicklung kann konzeptionell - so unser Argument - mit der rent gap-Theorie erklärt und damit die benannte Lücke in der Finanzialisierungs- und Gentrifizierungsforschung adressiert werden. Unser Beitrag zeigt, dass die rent gap-Theorie zur Erklärung von Dynamiken auf mittelstädtischen Immobilienmärkten produktiv ist, sofern diese sensibel für die lokal manifestierten Entstehungsbedingungen für Grundrentensteigerungen bleibt. Diese versteht Immobilieninvestitionen als Ausdruck der Verwertungslogiken des Kapitals, jedoch nicht als Automatismus, der sich an allen Orten gleichermaßen durchsetzt. Nicht umsonst betont Clark (2014), dass es Aufgabe guter Wohnungspolitik sei, auf ein „making rent gap theory not true“ hinzuwirken. Dies verweist bereits darauf, dass die konkreten Bedingungen vor Ort von großer Bedeutung dafür sind, dass sich eine Renditelücke auftut. Entsprechend ist die Entstehung von rent gaps nicht nur eine abstrakte Frage der Angebots- und Nachfrageentwicklung, sondern ihrer jeweiligen konkreten Manifestationen. So zeigt sich in den beiden Fallstudien grundsätzlich eine Steigerung der Nachfrage aufgrund des Bevölkerungswachstums, die aber aufgrund der verschiedenen Quantität und Qualität des Wachstums unterschiedlich ausfällt. In Fürstenwalde führt das - im Vergleich zu Neuruppin - geringere quantitative Bevölkerungswachstum nicht zur Öffnung einer Renditelücke, weil die Nachfragenden insgesamt nur eine geringe Zahlungskraft aufweisen und eine überregionale Nachfrage kaum generiert wurde. Anders verhält es sich in Neuruppin. Hier besteht aufgrund der lokal ansässigen Behörden etc. eine zahlungskräftige Nachfrage. Diese wird ergänzt durch ein stärkeres Bevölkerungswachstum, das unter anderem auf zahlungskräftigen Zuzug aus Berlin zurückzuführen ist, sowie das Auftreten (kapitalstärkerer) überregionaler Investoren. Angebotsseitig lässt sich für beide Fallstudien grundsätzlich eine Ausweitung des Wohnungsangebots durch Neubau konstatieren. In Fürstenwalde besteht kurzfristig die Möglichkeit, das Angebot durch Reaktivierung von Leerständen auszuweiten. In Neuruppin gibt es hingegen kaum Leerstand, der kurzfristig dem Markt zugeführt werden könnte. Angebotsseitig wird an den Fallstudien auch die Bedeutung der Stadtpolitik für rent gap-Steigerungen deutlich. So richtet sich die Veräußerungspolitik der Stadt Fürstenwalde an lokal ansässige Investoren sowie Nachfragende und hat damit eine preisdämpfende Wirkung. Etwas anders verhält es sich in Neuruppin. Auch hier erfolgt eine Ausweisung von Neubauflächen, angesichts früherer Schrumpfungsprognosen setzt man allerdings auf Innenentwicklung und ist mit Neuausweisungen zurückhaltend. Lokalen Projektent- wicklern gelingt es in Neuruppin darüber hinaus, Flächen zu entwickeln bzw. ein hochpreisiges neues Wohnungsangebot zu schaffen und durch aufwändige Vermarktung an überregionale Investoren zu veräußern.

Der empirische Fokus auf kleine Städte macht ein weiteres Problem sichtbar, welches weitere Untersuchungen vor Herausforderungen stellt. Entwicklungen in solchen Städten können nur schwer mit den etablierten Methoden aus der Forschung in Großstädten untersucht werden. Einerseits fallen erkennbare Aufwertungsprozesse in Mittelstädten (bislang) nicht so deutlich wie in Metropolen aus. Andererseits erweist sich die Datenverfügbarkeit als problematisch, da insbesondere auf kleinräumiger Ebene viele Daten nicht verfügbar sind. Folglich sollten auch die Methoden zur Operationalisierung der rent gap-Theorie und Gentrifizierungsforschung für kleinstädtische Kontexte weiterentwickelt bzw. ergänzt werden. Hier zeigen beide Fallstudien deutlich, dass eine Erweiterung statistischer Untersuchungen um qualitative Forschung äußerst fruchtbar ist, um immobilienwirtschaftliche Aufwertungsprozesse zu verstehen.

Angesichts des sich in einzelnen wachsenden Mittelstädten abzeichnenden Beginns immobilienwirtschaftlicher Aufwertungsprozesse stellen sich Fragen hinsichtlich einer langfristig sichergestellten sozial-gerechten Wohnraumversorgung. Ein gezieltes raumwissenschaftliches Monitoring der Wohnimmobilienmarktentwicklungen in diesen Städten mit Hilfe von unterschiedlichen Ansätzen der heterodoxen Wirtschaftsgeographie kann somit auch dazu beitragen, stadtpolitischen Entscheidungsträgern Erklärungsansätze für die jeweiligen lokalen Entwicklungen bereitzustellen. Darauf aufbauend können politische Stakeholder lokal angepasste Handlungsstrategien zur Steuerung eines ausgewogenen mittelstädtischen Wohnraumangebots entwickeln.

Danksagung: Wir danken Hans-Dieter von Frieling und Sebastian Schipper für die kritische Durchsicht früherer Versionen dieses Beitrags sowie den drei anonymen Gutachterinnen und Gutachtern für die wichtigen Hinweise zur Verbesserung des Manuskriptes. Die Forschungen zu Neuruppin wurden zum Teil im Rahmen des Projektes „Forschungsorientiertes Lehren und Lernen“ durch die Georg-August-Universität Göttingen, das Niedersächsische Ministerium für Wissenschaft und Kultur sowie das Bundesministeriums für Bildung und Forschung gefördert. Wir danken den Studierenden, die an dem Forschungsseminar mitgewirkt haben. Der Beitrag wurde publiziert mit Unterstützung der Alpen-Adria-Universität Klagenfurt. 


\section{Literaturverzeichnis}

Acxiom (Hg.) (2019): Kaufkraft 2019 in Deutschland. Online verfügbar unter https://www.acxiom.de/geomarketing/ kaufkraft-2019/, zuletzt geprüft am 18.12.2019.

AK OGA (2019): Immobilienmarktbericht Deutschland 2019 der Gutachterausschüsse in der Bundesrepublik Deutschland. Bonn: Bundesamt für Bauwesen und Raumordnung.

Amt für Statistik Berlin-Brandenburg (Hg.) (2020): Bevölkerungsstatistik für Berlin und Brandenburg. Online verfügbar unter www.statistik-berlin-brandenburg.de, zuletzt geprüft am 31.07.2020.

Atkinson, Rowland; Bridge, Gary (2005): Introduction. In: Rowland Atkinson und Gary Bridge (Hg.): Gentrification in a Global Context. The New Urban Colonialism. London [u. a.]: Routledge, S. 1-17.

B.B.S.M. (2018): Stadtumbau in der Zweiten Reihe. Städtebauliche Zielplanung der Stadt Fürstenwalde/Spree. Hg. v. Stadt Fürstenwalde/Spree. Online verfügbar unter http://binfo. fuerstenwalde-spree.de/getfile. php?id=37900\&type $=$ do\&, zuletzt geprüft am 09.09.2018.

Barnes, Trevor J.; Sheppard, Eric (2010): 'Nothing includes everything': towards engaged pluralism in Anglophone economic geography. In: Progress in Human Geography 34 (2), S. 193-214.

Behnke, Joachim; Baur, Nina; Behnke, Nathalie (2010): Empirische Methoden der Politikwissenschaft. 2. Aufl. Stuttgart: UTB.

Belina, Bernd (2011): Kapitalistische Raumproduktionen und ökonomische Krise. Zum Begriff des spatial fix bei David Harvey. In: Zeitschrift für Wirtschaftsgeographie 55 (4), S. 239-252.

Belina, Bernd (2017): Kapitalistischer Wohnungsbau: Ware, Spekulation, Finanzialisierung. In: Barbara Schönig, Justin Kadi und Sebastian Schipper (Hg.): Wohnraum für alle?! Bielefeld: Transcript Verlag, S. 31-45.

Beran, Fabian; Nuissl, Henning (2019): Verdrängung auf angespannten Wohnungsmärkten. Das Beispiel Berlin. Unter Mitarbeit von Stefan Krämer. Ludwigsburg: Wüstenrot Stiftung.

Bitterer, Nadine; Heeg, Susanne (2015): Die Macht der Zahlen. Kalkulative Praktiken in der Immobilienwirtschaft. In: Zeitschrift für Wirtschaftsgeographie 59 (1), S. 34-50.

Bohnsack, Ralf (2014): Rekonstruktive Sozialforschung. Einführung in qualitative Methoden. 9. Aufl. Opladen: Budrich (8242).

Christophers, Brett (2012): Anaemic Geographies of Financialisation. In: New Political Economy 17 (3), S. 271-291.

Clark, Eric (2014): Good urban governance: making rent gap theory not true. In: Geografiska Annaler: Series B, Human Geography 96 (4), S. 392-395.

Deutsche Bahn (2020): Fahrplanauskunft, zuletzt geprüft am 31.07.2020.

Döring, Christin; Ulbricht, Klaus (2016): Gentrification-Hotspots und Verdrängungsprozesse in Berlin. Eine quantitative Analyse. In: Ilse Helbrecht (Hg.): Gentrifizierung in Berlin. Verdrängungsprozesse und Bleibestrategien. Bielefeld: transcript (Urban Studies), S. 17-43.

Dutton, Paul (2005): Outside the Metropole: gentrification in provincial cities or provincial gentrification? In: Rowland Atkinson und Gary Bridge (Hg.): Gentrification in a Global Context. The New Urban Colonialism. London [u. a.]: Routledge, S. 214-229.
Empirica-Systeme Marktdatenbank (2020): Forschungsdatensatz.

Ernst Basler + Partner (2013): Regionales Standortentwicklungskonzept (STEK). FreiRaum Ruppiner Land Regionaler Wachstumskern Neuruppin und Kooperationsraum FreiRaum Ruppiner Land. Fontanestadt Neuruppin. Online verfügbar unter https://www.freiraumruppiner-land.de/fileadmin/freiraum/dateien/Downloads/ Regionales_Standortentwicklungskonzept_fuer_den_ RWK_Neuruppin_-_08.10.2013_01.pdf, zuletzt geprüft am 06.11.2019.

F+B (2020): Regionale Mieten und Preisentwicklung in Deutschland. Ergebnisse des F+B-Wohn-Index Deutschland Q4/2019. Hamburg. Online verfügbar unter https://www.f-und-b.de/ files/fb/content/Dokumente/News/F+B-Wohn-Index $\% 20$ 4-19\%20Quartalsbericht.pdf, zuletzt geprüft am 22.01.2020.

Fehlberg, Thorsten; Mießner, Michael (2015): Mietpreissteigerungen und Wohnungsengpässe abseits der Ballungsräume. Investitionen in Wohnimmobilien in B-Lagen und Regionalzentren - das Beispiel Göttingen. In: sublurban. zeitschrift für kritische stadtforschung 3 (1), S. 25-44.

Fernandez, Rodrigo; Aalbers, Manuel B. (2016): Financialization and housing: Between globalization and Varieties of Capitalism. In: Competition \& Change 20 (2), S. 71-88.

Fontanestadt Neuruppin (2017): Strategische Wohnbauflächenentwicklung. Wohnungsbau- und Innenentwicklungsstudie. Neuruppin.

Förste, Daniel; Bernt, Matthias (2016): Black Box Verdrängung: Bleiben im Kiez oder Wegzug an den Rand? Kleinräumige Wanderungen im Zuge von Aufwertungsprozessen in BerlinPrenzlauer Berg. In: Ilse Helbrecht (Hg.): Gentrifizierung in Berlin. Verdrängungsprozesse und Bleibestrategien. Bielefeld: transcript (Urban Studies), S. 45-67.

Gemeinsame Landesplanungsabteilung Berlin-Brandenburg (2018): Raumordnungsbericht 2018. Hauptstadtregion BerlinBrandenburg. Potsdam.

Gemeinsame Landesplanungsabteilung Berlin-Brandenburg ( $\mathrm{Hg}$.) (2019): Landesentwicklungsplan Hauptstadtregion BerlinBrandenburg (LEP HR). Potsdam.

GRUPPE PLANWERK (2018): Fürstenwalde 2030! Integriertes Stadtentwicklungskonzept (INSEK) der Stadt Fürstenwalde/ Spree. Hg. v. Stadt Fürstenwalde/Spree. Fürstenwalde.

Gutachterausschuss für Grundstückswerte im Landkreis Havelland (2019): Grundstücksmarktbericht 2018. Nauen.

Gutachterausschuss für Grundstückswerte im Landkreis Oder-Spree und in der Stadt Frankfurt (Oder) (Hg.) (2019): Grundstücksmarktbericht 2018. Landkreis Oder-Spree und Stadt Frankfurt (Oder). Beeskow.

Gutachterausschuss für Grundstückswerte im Landkreis OstprignitzRuppin (2019): Grundstücksmarktbericht 2018. Landkreis Ostprignitz-Ruppin. Neuruppin.

Gutachterausschuss für Grundstückswerte im Landkreis TeltowFläming (2019): Grundstücksmarktbericht 2018. Luckenwalde.

Gutachterausschuss für Grundstückswerte in der Stadt Brandenburg an der Havel (2019): Grundstücksmarktbericht $2018 \mathrm{der}$ kreisfreien Stadt Brandenburg an der Havel. Brandenburg an der Havel.

Gutachterausschuss für Grundstückswerte in der Stadt Cottbus (2019): Grundstücksmarktbericht 2018 Stadt Cottbus. Cottbus.

Harvey, David (2006 [1982]): The Limits to Capital. London [u. a.]: Verso. 
Heeg, Susanne (2004): Mobiler Immobilienmarkt? Finanzmarkt und Immobilienökonomie. In: Zeitschrift für Wirtschaftsgeographie 48 (2), S. 124-137.

Heeg, Susanne (2013): Wohnungen als Finanzanlage. Auswirkungen von Responsibilisierung und Finanzialisierung im Bereich des Wohnens. In: sublurban. zeitschrift für kritische stadtforschung (1), S. 75-99.

Hierse, Lin; Nuissl, Henning; Beran, Fabian; Czarnetzki, Felix (2017): Concurring urbanizations? Understanding the simultaneity of sub- and re-urbanization trends with the help of migration figures in Berlin. In: Regional Studies, Regional Science 4 (1), S. 189-201.

Holm, Andrej (2011): Wohnung als Ware. Zur Ökonomie und Politik der Wohnungsversorgung. In: Widersprüche 31 (121), S. 9-20.

Institut für Wohnen und Stadtentwicklung (2019): Wohnungsmarktstudie für die Fontanestadt Neuruppin. Ergebnisbericht 2019. Hg. v. Fontanestadt Neuruppin. Neuruppin.

IVD (2018): IVD-Wohn-Preisspiegel 2018/2019. Hg. v. IVD. IVD. Berlin.

Krätke, Stefan (1991): Strukturwandel der Städte. Städtesystem und Grundstücksmarkt in der „post-fordistischen“ Ära. Frankfurt/ Main, New York: Campus.

Krehl, Angelika; Weck, Sabine (2020): Doing comparative case study research in urban and regional studies: what can be learnt from practice? In: European Planning Studies „28 (9), S. 1858-1876.

Kuethe, Todd H.; Pede, Valerien O. (2011): Regional Housing Price Cycles: A Spatio-temporal Analysis Using US State-level Data. In: Regional Studies 45 (5), S. 563-574.

Lamnek, Siegfried (2010): Qualitative Sozialforschung. 5. Aufl. Weinheim: Beltz.

Lees, Loretta (2006): Gentrifying down the urban hierarchy. 'The cascade effect' in Portland, Maine. In: David Bell und Mark Jayne (Hg.): Small cities. Urban experience beyond the metropolis. Abingdon, Oxon, UK, New York, N.Y: Routledge (Questioning cities series), S. 91-104.

López-Morales, Ernesto (2010): Gentrification by Ground Rent Dispossession: The Shadows Cast by Large-Scale Urban Renewal in Santiago de Chile. In: Int J Urban Reg Res 43 (7), 330-357.

Marx, Karl (1962 [1867]): Das Kapital. Kritik der politischen Ökonomie. Band I: Der Produktionsprozess des Kapitals. Berlin: Dietz (Marx-Engels-Werke, 23).

Marx, Karl (2003 [1893]): Das Kapital. Kritik der politischen Ökonomie, Band 3. Der Gesamtprozess der kapitalistischen Produktion. 15. Aufl. Berlin: Dietz (Marx-Engels-Werke, Band 25).

Mattissek, Annika; Pfaffenbach, Carmella; Reuber, Paul (2013): Methoden der empirischen Humangeographie. 2. Aufl. Braunschweig: Westermann (Das geographische Seminar).

Meen, Geoffrey (1999): Regional House Prices and the Ripple Effect: A New Interpretation. In: Housing Studies 14 (6), S. 733-753.

Mießner, Michael (2019): Studentifizierung: Sozialräumliche Verdrängung in deutschen Universitätsstädten. Das Beispiel Göttingen. In: Geographische Rundschau (10), S. 22-27.

Miessner, Michael (2021): Studentification in Germany: How investors generate profits from student tenants in Goettingen and the impacts on urban segregation. In: European Urban and Regional Studies „28 (2), S. 133-154.

Mösgen, Andrea; Schipper, Sebastian (2017): Gentrifizierungsprozesse im Frankfurter Ostend. Stadtpolitische
Aufwertungsstrategien und Zuzug der Europäischen Zentralbank. In: Raumforschung und Raumordnung 75 (2), S. $125-141$.

Musil, Robert (2019): Immobiliengeographie. Märkte - Akteure Politik. Braunschweig: Westermann.

Nijman, Jan (2007): Introduction-Comparative Urbanism. In: Urban Geography 28 (1), S. 1-6.

Oberer Gutachterausschuss für Grundstückswerte im Land Brandenburg (2018): Grundstücksmarktbericht für das Land Brandenburg 2017. Potsdam.

Ouma, Stefan; Bläser, Kerstin (2015): Räume der Kalkulation. Geographien der finanziellen Ökonomisierung. In: Zeitschrift für Wirtschaftsgeographie 59 (4), S. 214-229.

Peck, Jamie (2012): Economic geography: Island life. In: Dialogues in Human Geography 2 (2), S. 113-133.

Perrot, René (2019): Entwicklung der Einwohnerzahl der Fontanestadt Neuruppin. Hg. v. Fontanestadt Neuruppin. Neuruppin. Online verfügbar unter https://www. neuruppin.de/fileadmin/_processed_/8/1/csm_ Einwohnerentwicklung_1991_bis_2018_1938fb969a.png, zuletzt geprüft am 06.11.2019.

Porsche, Lars; Steinführer, Annett; Sondermann, Martin (Hg.) (2019): Kleinstadtforschung in Deutschland. Stand, Perspektiven und Empfehlungen. Akademie für Raumforschung und Landesplanung. Hannover: Verlag der ARL (Arbeitsberichte der ARL, 28).

Porter, Michael (2010): The Rent Gap at the Metropolitan Scale: New York City's Land-Value Valleys, 1990-2006. In: Urban Geography 31 (3), S. 385-405.

Prima Gruppe (2019): Hauptseite. Online verfügbar unter https:// prima-gruppe.com/, zuletzt geprüft am 27.11.2019.

Reimann, Bettina; Kirchhoff, Gudrun; Pätzold, Ricarda; Strauss, Wolf-Christian (Hg.) (2018): Vielfalt gestalten. Integration und Stadtentwicklung in Klein- und Mittelstädten. Difu. Berlin: Difu (Edition Difu - Stadt Forschung Praxis, 17).

Rottke, Nico B. (2017): Besonderheiten von Immobilien und deren Märkten. In: Nico B. Rottke und Michael Voigtländer (Hg.): Immobilienwirtschaftslehre - Ökonomie. Wiesbaden: Springer Fachmedien, S. 83-100.

Sablowski, Thomas (2011): Krise und Kontinuität des finanzdominierten Akkumulationsregimes. In: Zeitschrift für Wirtschaftsgeographie 55 (1-2), S. 50-64.

Sakarya, Ipek; Başaran Uysal, Arzu (2018): Rural gentrification in the North Aegean Countryside (Turkey). In: ICONARP 6 (1), S. $99-125$.

Savills (2018): Investment Market Monthly: Germany. Savills Research Deutschland. Berlin u. a.

Scheuplein, Christoph (2020): Finanzialisierung in Ostdeutschland. In: Sören Becker und Matthias Naumann (Hg.): Regionalentwicklung in Ostdeutschland. Dynamiken, Perspektiven und der Beitrag der Humangeographie. 1. Aufl. Berlin: Springer Spektrum, S. 111-122.

Schipper, Sebastian (2013): Global-City-Formierung, Gentrifizierung und Grundrentenbildung in Frankfurt am Main. In: Zeitschrift für Wirtschaftsgeographie 57 (1-2), S. 185-200.

Schürt, Alexander (2013): Wohnungsengpässe und Mietensteigerungen. Aktuelle Mietentwicklungen in den Städten und Regionen. Bonn: BBSR (BBSR-Analysen Kompakt, 07/2013).

Seetor Residenz Neuruppin (2015): Leben am Wasser. Leben (wie) im Urlaub. Neuruppin. 
Sept, Ariane (2018): Zur Fallauswahl im qualitativen Stadtvergleich: eine Heuristik am Beispiel des Städtenetzwerks Cittaslow. In: Europa Regional 25.2017 (1), S. 2-14.

Sheppard, Eric (2018): Heterodoxy as Orthodoxy. Prolegomenon for a Geographical Political Economy. In: Gordon L. Clark, Maryann P. Feldman, Meric S. Gertler und Dariusz Wójcik (Hg.): The new Oxford handbook of economic geography. 1. Aufl. Oxford: Oxford University Press, S. 159-178.

Simons, Harald; Weiden, Lukas; Beuchert, Julian (2016): Abwanderung aus Nordwestbrandenburg. Folgen und strategische Lösungen. Hg. v. Empirica. Forschung und Beratung. Berlin.

Slater, Tom (2017): Planetary Rent Gaps. In: Antipode 49 (S1), S. 114-137.

Smith, Neil (1996): The new urban frontier. Gentrification and the revanchist city. London, New York: Routledge.

Smith, Neil (2006): Gentrification Generalized. In: Melissa S. Fisher, Greg Downey, Douglas R. Holmes und George E. Marcus (Hg.): Frontiers of Capital: Duke University Press, S. 191-208.

Smith, Neil (2010 [1984]): Uneven development. Nature, capital, and the production of space. 3. Aufl. London: Verso.

Smith, Neil (2019 [1979]): Für eine Theorie der Gentrifizierung. In: sublurban. zeitschrift für kritische stadtforschung 7 (3), S. 65-86.

Stadt Fürstenwalde (Hg.) (2019a): Kurzportrait. Online verfügbar unter http://www.fuerstenwalde-spree.de/seite/159549/ kurzportrait.html, zuletzt geprüft am 17.12.2019.
Stadt Fürstenwalde (Hg.) (2019b): Zahlen und Fakten rund um Fürstenwalde. Online verfügbar unter http://www. fuerstenwalde-spree.de/seite/162133/zahlen_und_fakten_ rund_um_fuerstenwalde.html, zuletzt geprüft am 17.12.2019.

sub\urban. zeitschrift für kritische stadtforschung (Hg.) (2019). subl urban. zeitschrift für kritische stadtforschung 7 (3).

TAG Immobilien AG (2018): Wohnungsmarktbericht Ostdeutschland 2017. TAG Immobilien AG. Hamburg.

Vornholz, Günter (2015): Internationale Immobilienökonomie. Globalisierung der Immobilienmärkte. Berlin: De Gruyter.

Ward, Callum; Aalbers, Manuel B. (2016): Virtual special issue editorial essay: 'The shitty rent business': What's the point of land rent theory? In: Urban Studies 53 (9), S. 1760-1783.

Wijburg, Gertjan; Aalbers, Manuel B. (2017): The alternative financialization of the German housing market. In: Housing Studies 32 (7), S. 968-989.

Zeller, Christian (2003): Bausteine zu einer Geographie des Kapitalismus. In: Zeitschrift für Wirtschaftsgeographie 47 (1), S. 5.

Zeller, Christian (2011): Verschiebungen der Krise im globalen Rentierregime. Ungleichgewichte und Suche nach neuen Feldern. In: Zeitschrift für Wirtschaftsgeographie 55 (1-2), S. 65-83. 